\title{
Fundus autofluorescence in age-related macular disease imaged with a laser scanning ophthalmoscope
}

\author{
Struharova K, Cernak M \\ Ophthalmic Department, University Hospital Cyril and Metod, Bratislava, Slovakia. katystruhar@gmail.com
}

\begin{abstract}
Age-related macular degeneration (ARMD) as the most common cause of legal blindness in industrialized countries remains an incompletely understood, complex retinal disease. Prophylactic and therapeutic options are still limited. Sensitive diagnostic tools and prognostic markers to evaluate disease stage and progression in the individual patient are needed. The retinal pigment epithelium (RPE) plays a key role in the disease process both in early and late variants of AMD. An excessive accumulation of lipofuscin granules in the lysosomal compartment of RPE cells represents a common downstream pathogenetic pathway in various retinal diseases including AMD. Fundus autofluorescence (FAF) imaging allows the visualization of the topographic distribution of lipofuscin over large retinal areas (Fig. 3, Ref. 13). Full Text in PDF www.elis.sk.

Key words: fundus autofluorescence, lipofuscin, retinal pigment epithelium (RPE), age-related macular degeneration (ARMD), geographic atrophy, confocal laser scan ophthalmoscopy (cSLO).
\end{abstract}

Fundus autofluorescence (FAF) imaging allows the visualization of the topographic distribution of lipofuscin over large retinal areas and therefore mapping of metabolic changes at the level of the RPE cell monolayer. Fundus autofluorescence (FAF) imaging using the confocal scanning laser ophthalmoscope (cSLO) was initially described by von Rückmann et al in 1995 (1). Pioneering work on the spectral analysis of the origin of the autofluorescence signal was performed by Delori and others in parallel (2). In 2003, Spaide introduced a modified fundus camera (using longer wavelengths than originally applied) for FAF imaging and, recently, the near-infrared autofluorescence imaging was described $(3,4$, 5). Various studies on FAF imaging have been performed in the meantime and this imaging technique is being increasingly used for research purposes. Today, FAF imaging is at the doorstep to a broader application as a noninvasive clinical imaging tool.

\section{Autofluorescence: imaging without dye}

This is the concept of using laser wavelengths to excite naturally occurring fluorophores in the retinal pigment epithelium (RPE) cells to obtain images without using any invasive dye. While autofluorescence is not a new imaging modality, it is the one we are beginning to understand and appreciate its potential.

Ophthalmic Department, University Hospital Cyril and Metod, Bratislava, Slovakia.

Address for correspondence: K. Struharova, MD, Ophthalmic Department, University Hospital Cyril and Metod, Slovakia, Antolska 11, SK85107 Bratislava, Slovakia.

Phone/Fax: +421.2.68672039

\section{What is fundus autofluorescence}

Fundus autofluorescene imaging is an in-vivo imaging method for metabolic mapping of naturally occurring fluorophores of the ocular fundus. The dominant source are fluorophores, such as A2-E (N-retinylidene-N-retinylethanolamine) in lipofuscin, that accumulate in the retinal pigment epithelium (RPE) as a by-product from an incomplete degradation of photoreceptor outer segments $(2,6$, 7). Previous studies suggest that lipofuscin and its constituent A2E may exert toxic effects on normal RPE cellular processes (5-10).

The topographic distribution of FAF intensities is altered in the presence of an excessive accumulation or loss of lipofuscin/RPE cells. Additional intrinsic fluorophores may occur in disease in the outer retina and the subneurosensory space. Minor fluorophores, such as collagen and elastin in choroidal blood vessel walls, may become visible secondary to RPE atrophy. Pathological alterations in the inner retina at the central macula, where the FAF signal is usually partially masked by luteal pigment (lutein and zeaxanthin), may result in manifest variations in FAF intensities.

Changes in fundus autofluorescence have been reported in patients with age-related macular degeneration (ARMD) (1-5).

Fundus autofluorescence has been also studied in various fundus diseases associated with retinal pigment epithelium (RPE) changes: serous RPE detachment $(4,5)$, the RPE tear $(2,5)$, the idiopathic macular hole (6), central serous chorioretinopathy (7), syphilitic posterior placoid chorioretinopathy (8), and macular dystrophies including adult vitelliform dystrophy, fundus flavimaculatus, Best disease, and dominant inherited pattern dystrophy ( 9 , 10). In patients with pseudoxanthoma elasticum (PXE), a number of changes in addition to angioid streaks have been reported in the retinal pigment epithelium (RPE). These RPE changes include a 


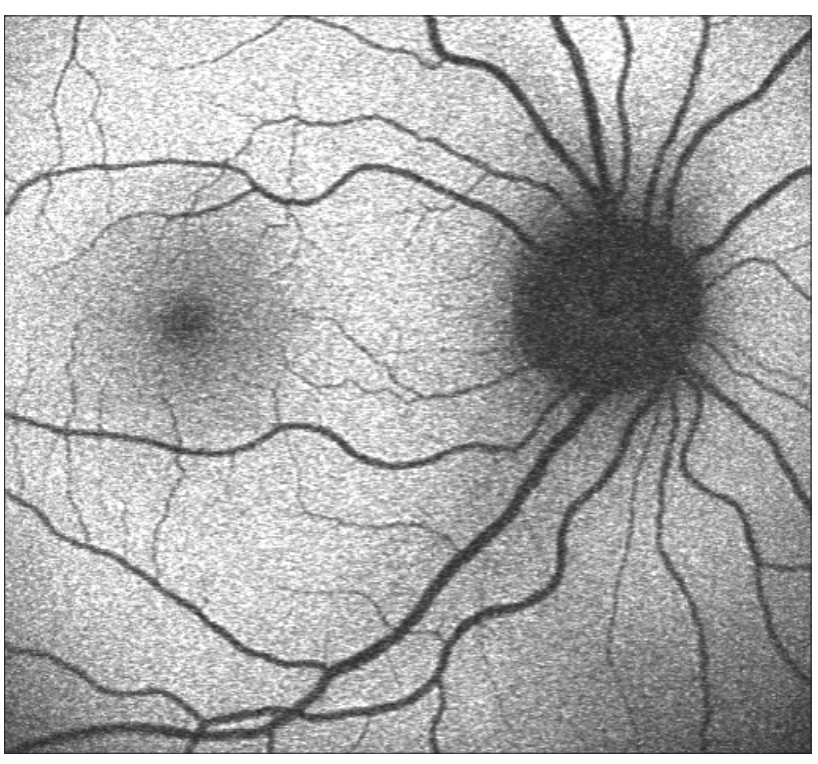

Fig. 1. Distribution of autofluorescence in a healthy eye $\left(\mathrm{HRA}, 30^{\circ}\right)$.

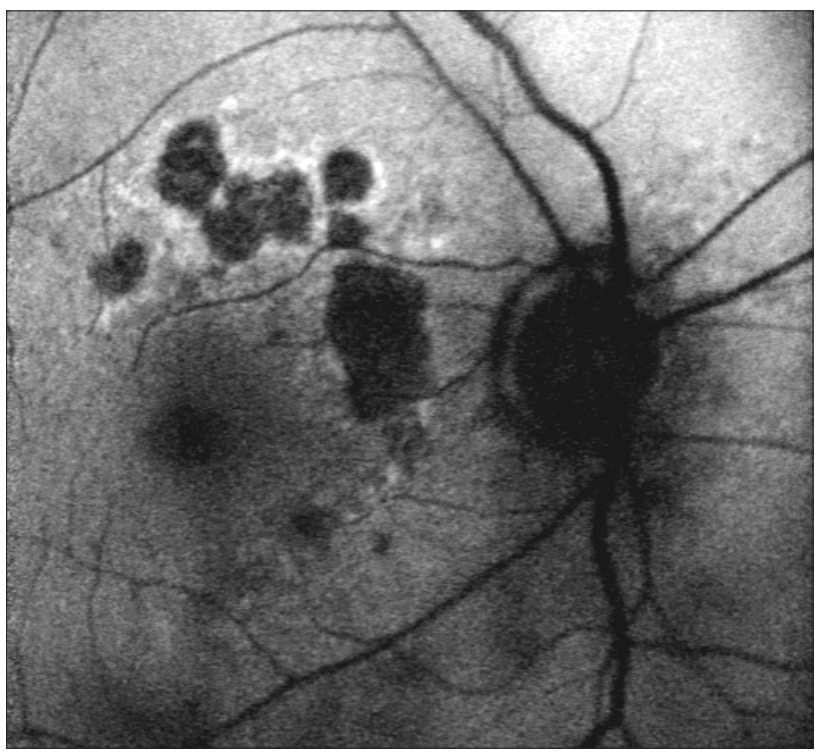

Fig. 2. Distribution of autofluorescence in a geographic atrophy (HRA, mean, $30^{\circ}$ ).

mottled appearance (called peau d'orange) (11-13), drusen-like spots (14-18), and salmon patches (areas of drusen-like spots and atrophy) $(19,20)$.

Autofluorescence images, which are obtained through the use of confocal laser scanning ophthalmoscopy (cSLO), play an increasing role in the understanding of drusen and age-related macular degeneration (ARMD). An example of the typical autofluorescence image in a normal retina can be seen in the Figure 1. An excessive lipofuscin accumulation in the RPE, as imaged by focally increased autofluorescence (FIAF), has been proposed to be a marker of RPE disease and photoreceptor cell degeneration (5, 6). The presence of large, soft drusen without atrophy, regardless

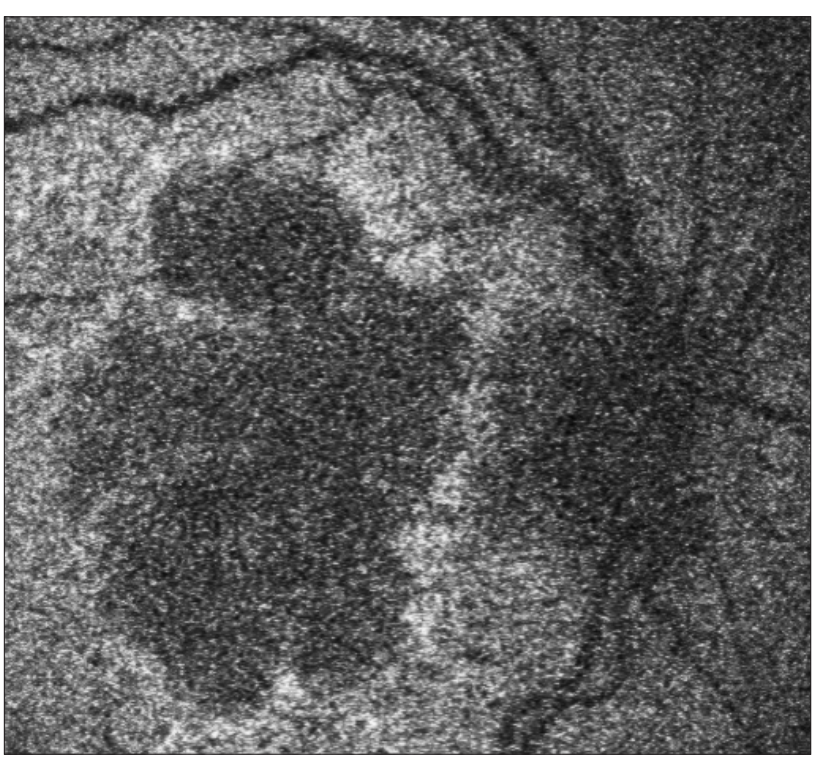

Fig. 3. Autofluorescence of a geographic atrophy (HRA, 30 ${ }^{\circ}$ ).

of the presence or lack of pigment changes, was also associated with areas of increased autofluorescence (FIAF).

In patients with geographic atrophy (GA), FIAF is found mostly adjacent to drusen and areas of GA. The example of this can be seen in the Figures 2 and 3, which shows the eyes of the patients with some atrophic changes and GA. It is possible to see less co-localization of the areas of increased autofluorescence in the eye with more atrophy. Images with the evidence of choroidal neovascularization (CNV) were remarkable for an increased finding of reticular autofluorescence patterns (13). Focally decreased autofluorescence (FDAF), especially over larger areas, is equivalent to geographic atrophy (GA) (7). Correlations between macular pathological changes and AF imaging can be helpful in the staging and treatment of ARMD.

\section{Identification of early disease stages}

Fundus autofluorescence imaging may allow the identification of retinal diseases when they are not evident. Metabolic changes at the level of the photoreceptor/RPE complex may not be visualized by funduscopy or other routine imaging techniques. This is particularly helpful when examining patients with unknown visual loss or a positive familiar history of hereditary retinal diseases $(9,10)$.

\section{Phenotyping}

Alterations in FAF intensities at the outer retina in various retinal diseases are typically much more pronounced. This helps in diagnosing hereditary retinal disorders, such as Stargardt disease and vitelliform macular and pattern dystrophies. Focal flecks in Stargardt disease associated with increased FAF signal are more delineated on FAF images compared to fundus photographs (12) (Fig. 4). FAF findings can also help to distinguish the AMD from late onset of macular dystrophies mimicking the age-related changes. 


\section{Monitoring of disease progression}

Levels of increased FAF that do not or only poorly correlate with findings obtained by other imaging techniques. These FAF changes, remote from visible alterations, may suggest more widespread abnormalities and diseased retinal areas. Focally increased FAF and therefore an excessive RPE lipofuscin load may indicate dysfunctioning RPE cells. Areas with increased FAF signal and thus and excessive RPE lipofuscin accumulation precede the development of new areas or the enlargement of existing atrophic patches in geographic atrophy secondary to AMD. Extension of abnormal FAF has an impact on atrophy enlargement rates over time and may therefore serve as a predictive determinant. Due to the absence of RPE lipofuscin, atrophic areas such as in atrophic AMD or Stargardt disease, exhibit a markedly reduced signal. Such diseased areas and atrophic patches can be easily identified and, moreover, precisely quantified by customized image-analysis software. This allows the noninvasive monitoring of atrophy progression over time (Fig. 2).

\section{Conclusions}

FAF imaging gives information over and above conventional imaging techniques in various retinal disorders. It is easy to perform and it is a noninvasive imaging method. Particularly in disorders of the outer retina, the application offers the opportunity to identify disease-related abnormalities and to determine the integrity of the RPE. It represents not just a technique to visualize structural changes, but it actually allows the metabolic mapping and correlation with retinal function. Several studies have demonstrated its clinical relevance, while there are numerous promising future applications. In combination with other emerging technologies such as spectral-domain optical coherence tomography, FAF imaging may considerably contribute to our understanding of retinal disease.

\section{References}

1. Delori FC et al. In vivo fluorescence of the ocular fundus exhibits retinal pigment epithelium lipofuscin characteristics. Invest Ophthalmol Vis Sci 1995; 36: 718-729.

2. von Ruckmann A et al. In vivo fundus autofluorescence in macular dystrophies. Arch Ophthalmol 1997; 36: 718-729.

3. Kitigawa $\mathrm{K}$ et al. In vivo quantification of autofluorescence in human retinal pigment epithelium. Ophthalmologica 1989; 199: 116-121.

4. Weiter JJ et al. Retinal pigment epithelial lipofuscin and melanin and choroidal melanin in human eye. Invest Ophthalmol Vis Sci 1986; 27 : $145-152$.

5. Delori FC et al. Autofluorescence distribution associated with drusen in age-related macular degeneration. Invest Ophthalmol Vis Sci 2000; 41: 496-504.

6. Dorey $\mathbf{C}$ et al. Cell loss in the ageing retina: relationship to lipofuscin accumulation and macular degeneration. Invest Ophthalmol Vis Sci 1989; 30: 1691-1699.

7. Holz FG et al. Fundus autofluorescence and development of geographic atrophy in age-related macular degeneration. Invest Ophthalmol Vis Sci 2001; 42: 1051-1056.

8. Smith RT et al. A method of drusen measurement based on reconstruction of fundus reflectance. Br J Ophthalmol 2005; 89: 87-91.

9. Smith RT et al. Automated detection of macular drusen using geometric background leveling and threshold selection. Arch Ophthalmol 2005; 123: 200-207.

10. Smith RT et al. A method of drusen measurement based on the geometry of fundus reflectance. BioMed Eng Online 2003; 2: 10.

11. Smith RT et al. Autofluorescence characteristics of normal foveas and reconstruction of foveal reflectance. Br J Ophthalmol 2005; 89: 87-91.

12. Hwang JC et al. Predictive value of fundus autofluorescence for development of geographic atrophy in age-related macular degeneration. Invest Ophthal Vis Sci 2006; 47:2655-2661.

13. Smith RT et al. Autofluorescence characteristics of early, atrophic, and high-risk fellow eyes in age-related macular degeneration. Invest Ophthalmol Vis Sci 2006; 47 (12): 5495-5504. 